\title{
Dual Healthcare System Use is Associated with Higher Mortality in Heart Failure
}

\author{
R. Neal Axon, MD, MSCR ${ }^{1,2}$, Mulugeta Gebregziabher, $\mathrm{PhD}^{1,3}$, Charles J. Everett, $\mathrm{PhD}^{1}$, Paul Heidenreich, $\mathrm{MD}$, \\ $\mathrm{MS}^{4,5}$ \& Kelly J. Hunt, $\mathrm{PhD}^{1,3}$ \\ ${ }^{1}$ Charleston Health Equity and Rural Outreach Innovation Center (HEROIC), Ralph H. Johnson VA Medical \\ Center, Charleston, SC, USA \\ ${ }^{2}$ Division of General Internal Medicine, Department of Medicine, The Medical University of South Carolina, \\ Charleston, SC, USA \\ ${ }^{3}$ Department of Public Health Sciences, The Medical University of South Carolina, Charleston, SC, USA \\ ${ }^{4}$ Division of Cardiology, VA Palo Alto Healthcare System, USA \\ ${ }^{5}$ Division of Cardiology, Stanford University Medical Center, USA \\ Correspondence: R. Neal Axon, MD, MSCR, 109 Bee Street, Mail Code 111, Charleston, SC 29401, USA. \\ 1-(843)-577-5011 ext. 5526. E-mail: axon@musc.edu
}

Received: June 18, 2017 Accepted: July 8, 2017 Online Published: September 20, 2017

doi:10.5539/gjhs.v9n12p9 URL: https://doi.org/10.5539/gjhs.v9n12p9

\begin{abstract}
Background: Heart failure is associated with high mortality, and health system-related factors contribute to this risk. Dual health system use occurs when patients receive care from multiple facilities over time, and such fractured care has been associated with higher healthcare utilization and higher mortality in selected conditions.

Methods: We analyzed a cohort of 13,948 U.S. Veterans receiving emergency department (ED) or hospital care for heart failure between 2007-2011 using information from the VA, Medicare, and an all-payor state-level claims database. Cox proportional hazards regression was used to model the association between all-cause mortality and dual use comparing dual users to those receiving VA-only care or non-VA only care.

Results: In fully adjusted models accounting for age, gender, race/ethnicity, marital status, disability, and comorbidities, dual use Veterans with heart failure had higher hazard for mortality from their date of entry into the cohort (HR 1.21, 95\% CI 1.11, 1.32, $<<0.0001$ ) and from the date of their last hospitalization (HR 1.40, 95\% CI $1.28-1.53, \mathrm{p}<0.0001)$ as compared to VA-only users. Non-VA only users did not have significantly different hazard for mortality compared to VA-only users. Additional models in a subset of patients which also included laboratory data for brain-type natriuretic peptide, blood urea nitrogen, and serum sodium yielded similar results.
\end{abstract}

Conclusions: Dual use appears to be associated with higher risk for mortality among Veterans with heart failure. While cross-system care is necessary and even desirable in many situations, strategies to identify high-risk patients and to mitigate risks of fractured care are warranted.

Keywords: heart failure, healthcare utilization, health care seeking behavior, hospitalization, mortality

\section{Introduction}

Heart failure (HF) is a serious chronic medical illness which causes over 800,000 emergency department (ED) visits and over 1.1 million hospitalizations annually and is associated with $\$ 32$ billion in healthcare costs (Blecker, Ladapo, Doran, Goldfeld, \& Katz, 2014; Lloyd-Jones, Adams, \& M., 2008). HF is associated with significant morbidity including chronic dyspnea, fatigue, and decreased quality of life. Furthermore, patients hospitalized for HF have 30-day all-cause mortality rates of approximately $8.8 \%$, and HF patients have a 5 -year mortality rate that approaches 50\%. (Cheng et al., 2014; Roger et al., 2004)

An assortment of clinical factors have been associated with increased risk for death among HF patients, including age, New York Heart Association (NYHA) functional class, left ventricular ejection fraction, and comorbid medical conditions.(Adams et al., 2005; Chaudhry et al., 2013; David C. Goodman, Fisher, \& Chang, 2011; D. C. Goodman, Fisher, \& Chiang-Hua, February, 2013; Lupon, Vila, \& Bayes-Genis, 2015; Ross, Mulvey et al., 2008) Increasingly, system-related factors such as dual healthcare system use (dual use) have been associated with higher 
healthcare utilization and worse outcomes for several diseases. Dual use occurs when patients receive care from multiple providers or healthcare facilities. Within the Medicare population in the U.S., approximately $20 \%$ of patients re-hospitalized within 30 days after a HF hospital admission are admitted to different facilities on their second stay (Nasir et al., 2010). In the U.S. Veterans Health Administration, a majority of Veteran hospitalizations for cardiovascular diseases occur at non-VA facilities, especially among older Veterans over age 65 years (Weeks, West, Wallace, \& Fisher, 2008). A recent study found that Veterans with HF who were dual users of acute care had $15 \%$ higher adjusted rates of ED visits, $40 \%$ higher rates of hospital admission for $\mathrm{HF}$, and $46 \%$ higher rates of all-cause 30-day hospital readmission ("Blindd for Review,").

Concerns exist that dual healthcare system use may also be associated with higher risk of mortality (Ross, Keyhani, et al., 2008; Wolinsky, An, Liu, Miller, \& Rosenthal, 2007; Wolinsky et al., 2006). Jia and colleagues studied stroke patients cared for in VA and non-VA facilities, and they observed that dual use was associated with significantly higher rates of 12-month rehospitalization and death (Jia et al., 2007). Tarlov and colleagues studied colon cancer patients, and they found that a higher hazard of death across cancer stages for dual users as compared to single-system users (Tarlov et al., 2012). We hypothesized that because HF is a chronic condition prone to frequent exacerbation, dual users of acute care might face similar risks. The present study was designed to determine if dual use of acute care by Veterans with HF is associated with higher risk for death as compared to single-system users.

\section{Methods}

\subsection{Study Population}

We created a state-wide cohort of Veterans with HF by linking multiple patient and administrative files including: the Veterans Health Administration (VHA) corporate data warehouse available from VA National Data Systems; VA/Medicare files available from the Veterans Information Resource Center; and the South Carolina Office of Revenue and Fiscal Affairs (SC-RFA) as detailed in an earlier manuscript.("Blinded for Review,") This project was approved by the VA Central IRB as well as the Research and Development Committee at the Ralph H. Johnson VAMC.

Subjects for this analysis were selected from a larger cohort $(n=203,959)$ based on the following inclusion criteria for which included: enrollment for care in VHA; attendance at one or more primary care appointments during the study timeframe (2007-2011); and residence in the state of South Carolina $(\mathrm{n}=136,244)$. Subjects were considered to have HF if they had one or more inpatient or outpatient diagnosis codes for heart failure in a given year (ICD-9 402.01, 402.11, 402.91, 429.3x, 425.xx, 428.xx). The final analytic dataset $(\mathrm{n}=13,948)$ was comprised of patients with at least one ED visit or hospitalization for HF at a VA or non-VA facility during the study timeframe. Subjects were censored in the event of death, loss to follow up, or if still enrolled for VA care as of March, 2012.

\subsection{Outcome Measures}

The main outcome measure was time to death (all-cause death). Veterans were followed from two time points. For the first, Veterans were followed from time of entry (entry date) into the study until death, loss to follow-up, or through March 2012 (exit date). For the second, Veterans were followed from time of their last hospitalization (date of hospitalization) until death, loss to follow-up, or through March 2012 (exit date). To ensure adequate follow-up time for the second analysis to be included the date of the last hospitalization had to be on or before September 30, 2011. A subject was considered censored if alive by March 2012.

\subsection{Primary Covariate}

Dual use was defined at the patient level with patients being categorized based on where they received acute ED and/or hospital care as VA-only users, non-VA only users, and dual users.

\subsection{Other Covariates}

Additional covariates of interest included age, gender, race/ethnicity, marital status, service connected disability status, and comorbidities. We analyzed age as a continuous variable. Gender was categorized as male vs. female. Race/ethnicity, categories were non-Hispanic white (NHW), non-Hispanic black (NHB), Hispanic, and other/missing/unknown. Marital status categories were married, divorced, widowed, never married, unknown. Rurality categories were urban, rural, highly rural or missing. Service connected disability is an administrative determination that has been used as a proxy for disease burden and copayment in prior studies of Veterans. We categorized subjects as $\geq$ or $<50 \%$ service connected for disability.("Blinded for Review,") We used the Elixhauser classification system to capture comorbidities, and each diagnosis was classified as present or absent for a given patient.(Quan et al., 2005) Additionally, for Veterans with available laboratory measurements in VA, we examined 
brain natriuretic peptide (BNP) levels, blood urea nitrogen (BUN) levels, and serum sodium levels.

\subsection{Statistical Analysis}

In preliminary analyses, crude associations were examined between mortality and all measured covariates using chi-square tests for categorical variables and t-tests for continuous variables. Cox proportional hazards regression was used to model the association between all-cause mortality and dual use status with two index variables representing the three categories of dual use (i.e., VA care only, Non-VA care only and Dual Users).(Cox, 1972) Time to death was defined as the number of days from time of entry to the cohort to time of death or censoring (i.e., day last seen or March 2012). Appropriateness of the assumption of proportionality by examining log plots and by testing the coefficients of the interactions of $\log ($ time $)$ with the respective covariate in a multivariate analyses .(Lin, Wei, \& Ying, 1993) Our explanatory association models were fitted sequentially as follows. Initial models were unadjusted (Model 1), followed by a model adjusted for demographic characteristics (Model 2) and a final model adjusted for both demographic characteristics as well as comorbidities (Model 3). HR estimates with their corresponding $95 \%$ confidence interval $(95 \%$ CI) are reported. The Kaplan-Meier method was used to plot the survival functions for each dual use category. Residual analysis was used to assess the goodness of fit of each of the models. All data analyses were conducted using SAS 9.3 (SAS Institute, Inc., Cary, NC).

\section{Results}

Our final sample included 13,948 Veterans with HF with ED visits or hospitalizations during the study timeframe (Table 1). Survival probabilty over time from entry into the cohort is illustarted in Figure 1 while survival probability over time from date of last hospitalization is illustrated in Figure 2. Examining survival from entry into the cohort, survival estimates appear somewhat simlar until roughly 3 years into the follow-up period when survival estimates decline for both dual users and patients who recieved care only outside the VA when compared to patients who recieve care only within the VA (Figure 1). In contrast, survival estimates from time of last hospitalization quickly separate with dual users consistently having the lowest survial estimates and patients who recieve only within the VA consistently having the highest survial estimates within the VA (Figure 2). 
Table 1. Subject characteristics by dual use status

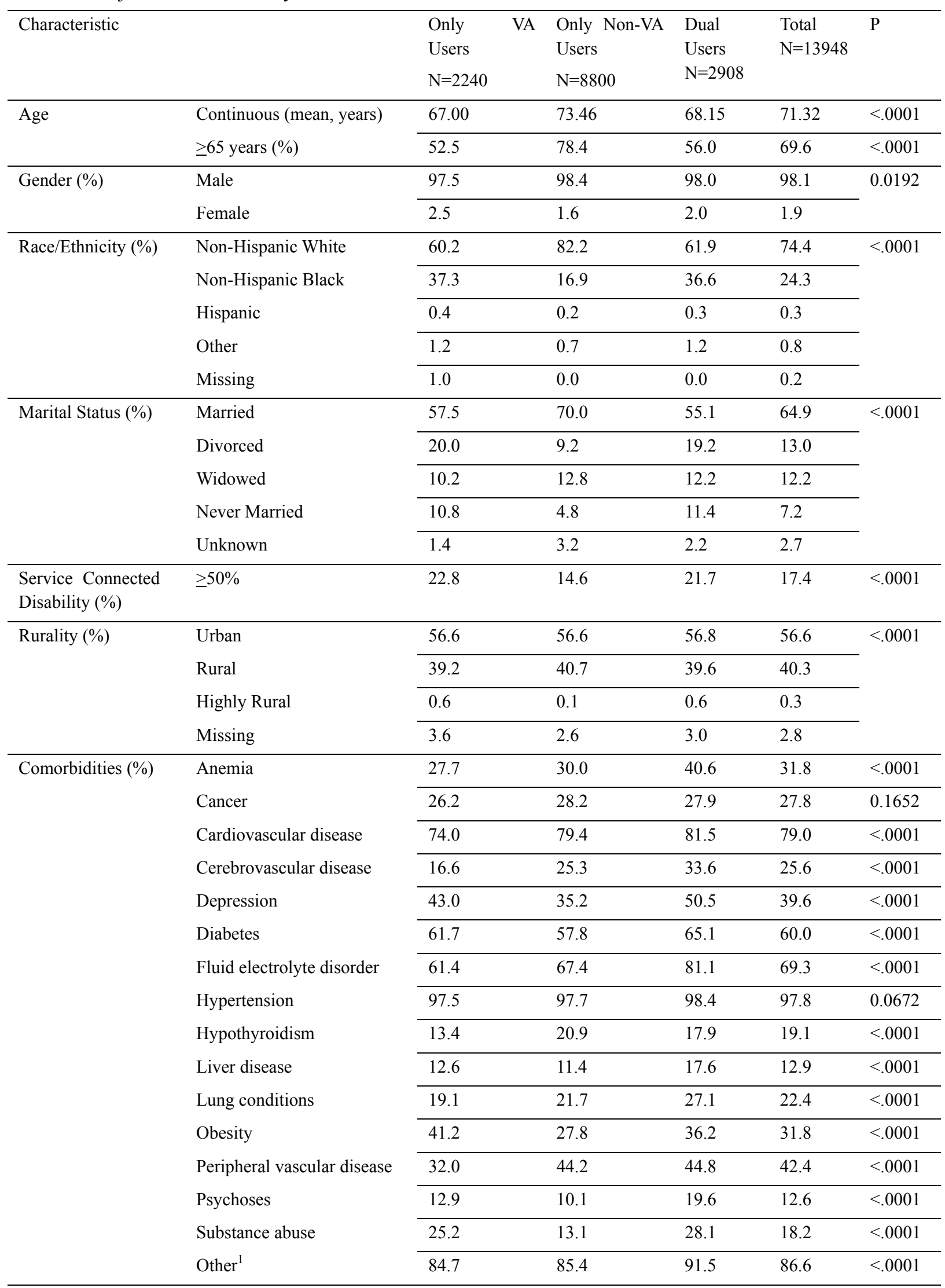

\footnotetext{
${ }^{1}$ Includes aids, chronic pulmonary disease, coagulopathy, peptic ulcer disease, renal failure, and rheumatoid arthritis.
} 


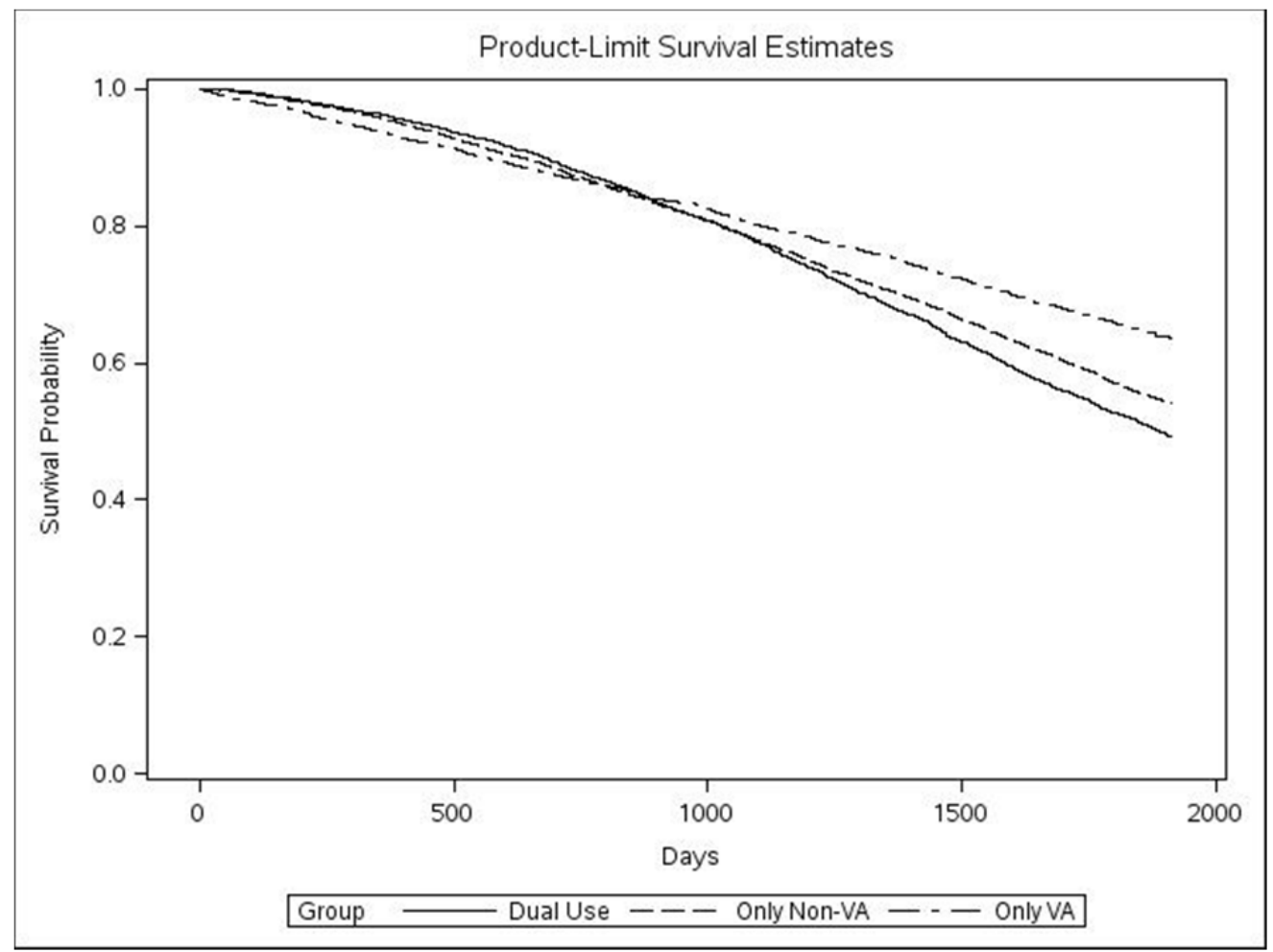

Figure 1. Survival probability over time from entry into the cohort

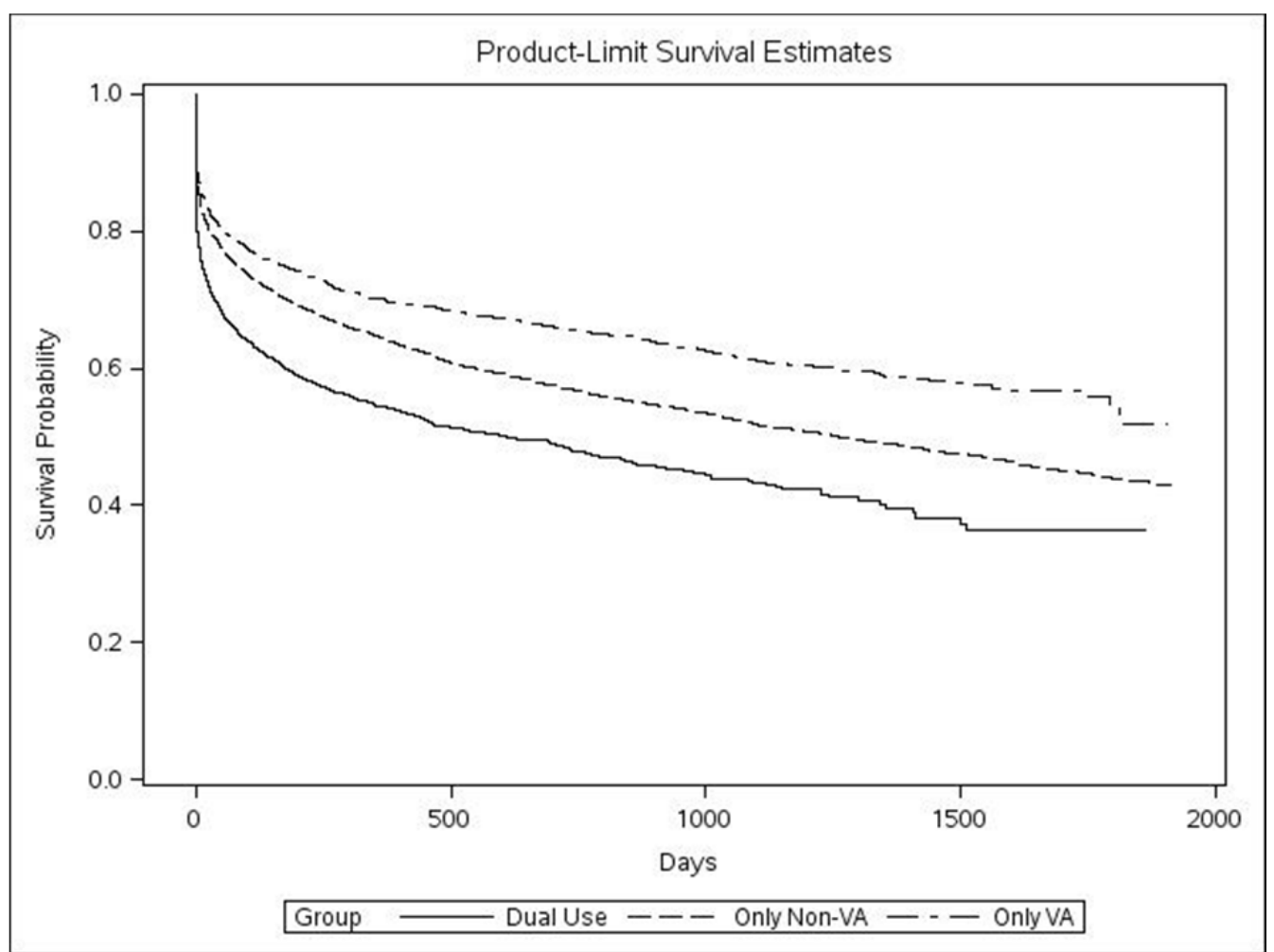

Figure 2. Survival probability over time from last hospitalization

Hazard ratios for the assocation between dual use and mortality using entry into the cohort as the staring time are presented in Table 2. In the unadjusted model dual users had 48\% increased hazard of mortality [1.48 (95\% CI: $1.36,1.61)$ and only non-VA users had $31 \%$ increased hazard of mortality $[1.31$ (95\% CI: $1.22,1.42)$ when 
compared to patients who recieved care only within the VA. After adjusting for age, gender, race/ethnicity, marital status and service disability dual users remained at $38 \%$ increased hazard of mortality [1.38 (95\% CI: 1.27, 1.51)] when compared to patients who received care only within the VA, while only non-VA users had similar hazard of mortailty when compared to patients who recieved care only within the VA [1.03 (95\% CI: 0.95, 1.12)]. Moreover, after further adjusting for comorbidity burden dual users remained at $21 \%$ increased hazard of mortality [1.21 (95\% CI: 1.11, 1.32)] relative to patients who recieved care only within the VA. Supplementary Table 1 includes hazard ratios for all variables included within model 3 reported in Table 2.

Table 2. Association between dual use and mortality: Time to death from entry into the cohort

\begin{tabular}{lllll}
\hline Model & Dual Use Status & Hazard Ratio & $95 \%$ CI & p-value \\
\hline 1 & Only VA Users & 1.00 & --- & $<.0001$ \\
\cline { 2 - 5 } & Only Non-VA Users & 1.31 & $1.22-1.42$ & $<.0001$ \\
\cline { 2 - 5 } & Dual Users & 1.48 & $1.36-1.61$ & 0.48 \\
\hline 2 & Only VA Users & 1.00 & -- & $<.0001$ \\
\cline { 2 - 5 } & Only Non-VA Users & 1.03 & $0.95-1.12$ & 0.31 \\
\cline { 2 - 5 } & Dual Users & 1.38 & $1.27-1.51$ & $<.0001$ \\
\hline
\end{tabular}

Model 1: Unadjusted

Model 2: Adjusted for Demographics (Age, Gender, Race/Ethnicity, Marital Status, Service Disability)

Model 3: Adjusted for Demographics, and Comorbidities (Cancer, Cerebrovascular Disease, Depression, Diabetes, Fluid Electrolyte Disorder, Hypertension, Hypothyroidism, Liver Disease, Lung Conditions, Obesity, Peripheral Vascular Disease, Other - Includes Aids, Chronic Pulmonary Disease, Coagulopathy, Peptic Ulcer Disease, Renal Failure, and Rheumatoid Arthritis)

Table 3. Association between dual use and mortality: Time to death from last hospitalization

\begin{tabular}{lllll}
\hline Model & Dual Use Status & Hazard Ratio & $95 \%$ CI & p-value \\
\hline 1 & Only VA Users & 1.00 & --- & $<.0001$ \\
\cline { 2 - 5 } & Only Non-VA Users & 1.29 & $1.20-1.40$ & $<.0001$ \\
\cline { 2 - 5 } & Dual Users & 1.75 & $1.60-1.91$ & 0.64 \\
\hline 2 & Only VA Users & 1.00 & --- & $<.0001$ \\
\cline { 2 - 5 } & Only Non-VA Users & 1.02 & $0.94-1.10$ & 0.15 \\
\cline { 2 - 5 } & Dual Users & 1.64 & $1.50-1.79$ & $<.0001$ \\
\hline
\end{tabular}

Model 1: Unadjusted

Model 2: Adjusted for Demographics (Age, Gender, Race/Ethnicity, Marital Status, Service Disability)

Model 3: Adjusted for Demographics, and Comorbidities (Cancer, Cerebrovascular Disease, Depression, Diabetes, Fluid Electrolyte Disorder, Hypertension, Hypothyroidism, Liver Disease, Lung Conditions, Obesity, Peripheral Vascular Disease, Other - Includes Aids, Chronic Pulmonary Disease, Coagulopathy, Peptic Ulcer Disease, Renal Failure, and Rheumatoid Arthritis)

Hazard ratios for the assocation between dual use and mortality using date from last hospitalization as the staring 
time are presented in Table 3. In the unadjusted model dual users had 75\% increased hazard of mortality [1.75 (95\% CI: $1.60,1.91)$ and only non-VA users had 29\% increased hazard of mortality [1.29 (95\% CI: $1.20,1.40)$ when compared to patients who recieved care only within the VA. After adjusting for age, gender, race/ethnicity, marital status and service disability dual users remained at 64\% increased hazard of mortality [1.64 (95\% CI: 1.50, 1.79)] when compared to patients who received care only within the VA, while only non-VA users had similar hazard of mortailty when compared to patients who recieved care only within the VA [1.02 (95\% CI: 0.94, 1.10)]. Moreover, after further adjusting for comorbidity burden dual users remained at $40 \%$ increased hazard of mortality [1.40 (95\% CI: $1.28,1.53)]$ relative to patients who recieved care only within the VA. Supplementary Table 2 includes hazard ratios for all variables included within the third model reported in Table 3. Among those who died prior to 180 days after the end of the study period, the median number of days from last hospitalziation to death was 11 in dual users, 45 in only non-VA users and 29 in patients who received care only within the VA, while the mean number of hospitalizations within 180 days of death was 2.34 in dual users, 1.35 in only non-VA users and 1.40 in patiens who received care only within the VA.

Supplementary Tables 3 and 4 present analyses limited to veterans who received at least some care within the VA (i.e., dual users and only VA users) further adjusting for clinical chemistries often related to mortality (i.e., BNP, BUN and sodium ). Results for the analysis using entry into the cohort as the starting time indicate dual users are at $21 \%$ increased hazard of mortality $[1.21(95 \% \mathrm{CI}: 1.08,1.36)]$ relative to patients who recieved care only within the VA (Supplementatry Table 3). Results for the analysis using date from last hospitalization as the starting time indicate dual users are at 34\% increased hazard of mortality [1.34(95\% CI: $1.20,1.51)]$ relative to patients who recieved care only within the VA (Supplementatry Table 4).

\section{Discussion}

The present study is perhaps the first to examine the association between dual health system use and mortality in a cohort of Veterans with HF with robust information available for both VA and non-VA acute healthcare utilization. We found that dual users had higher hazard for mortality whether measured from their entry into the study cohort (first ED visit or hospitalization) or from their final hospitalization during the study time period. These findings are important in helping to identify dual use as an independent risk factor for death in this population that is not traditionally considered in clinical and policy decision making.

There is relatively limited research in this area to date. Several prior studies have examined process-related quality comparing VA to non-VA care systems of care. (Asch et al., 2004; Keating et al., 2011; Trivedi \& Grebla, 2011) Few studies have assessed mortality risk across systems, though Selim and colleagues compared mortality risk for several conditions among VA patients compared to Medicare Advantage patients. (Selim et al., 2006) After controlling for baseline health status, sociodemographic factors, and comorbid conditions, VA patients had lower mortality risk overall, and HF patients specifically had lower hazard for mortality (HR 1.520, 95\% CI 1.497, 1.544). In contrast to this study, we did not observe differential hazard for mortality between VA-only and non-VA only patients, though we did not parse out specific non-VA payor groups. Even fewer studies have examined mortality specifically related to dual use. Our findings are similar to observations made in stroke patients and colon cancer patients, though. (Jia et al., 2007; Tarlov et al., 2012) For example, Jia and colleagues examined outcomes for VHA enrolled patients hospitalized for and discharged alive after acute stroke, and they observed that, compared to VHA-only patients, those receiving dual system care had higher odds for death at 12 months (adjusted odds ratio 1.6, 95\% CI 1.0, 2.4 for VA-Medicare users; and OR 2.8, 95\% CI 1.0-7.4 for VA-Medicaid users). (Jia et al., 2007) It is not clear whether similar observations apply to other cardiovascular diseases or chronic diseases, however.

It is important to consider the potential mediators of this phenomenon. First, it seems clear that a higher proportion of dual users tended to have certain comorbidities, and the total number of comorbidities for dual users was also higher in our sample. This observation may be somewhat artifactual, though, if diagnostic coding practices and underlying financial incentives for coding differ within and outside the VHA. Regardless, dual users may simply be sicker patients. If so, dual use could be viewed as an important factor associated with higher mortality risk. It is also possible that quality of HF care might vary across healthcare systems. On one hand, hospitals with the highest levels of adherence to Get With The Guidelines (GWTG) quality metrics did not have significantly lower mortality compared to other facilities participating in the GWTG program after controlling for patient and hospital level covariates.(Hernandez et al., 2010) On the other hand, studies of diabetes disease management have among dual users have shown instances of duplicative care and poorer glycemic control among Veterans, and similar process outcomes are plausible in HF. (Helmer et al., 2008; Maciejewski et al., 2013) Finally, it is possible that lapses in communication and data sharing, medication management, and timely follow up after hospitalizations for heart 
failure contributed to the observed differences in heart failure outcomes. If so, this would put dual use in the causal pathway for higher mortality, and strategies should be devised to directly address these issues.

In any event, strateties are needed that can begin to mitigate this risk. To the extent that dual use is an indicator of higher dissease burden, it could be used as a trigger to identify patients as candidates for more intensive heart failure management programs many of which are available in the VA. Where congruent with patient and family preferences, better identification of end-stage heart failure patients may also facilitate appropriate referrals for palliative and end-of-life care that could improve quality of life for patients. Strategies that promote cross-system communication may help to improve information exchange across health systems. In addition, the VA Office of Community Care is working on new care coordination programs designed to improve care across systems and to re-integrate Veterans into the VA healthcare system after episodes of community Care (Personal Communication, Leo Greenstone, MD). Future work should focus on better understanding these and other potential mechanims of this dual use phenomenon and on developing interventions to mitigate risk.

Our study is subject to certain limitations. First, as stated above, dual users had higher rates than all-VA users for several comorbidities including other cardiovascular disorders, cerebrovascular disease, diabetes, and peripheral vascular disease. Despite controlling for variations in these factors, our results may be subject to some residual confounding based on these and other unobserved factors. Second, our cohort included only $1.9 \%$ female subjects, so our findings related to women should be interpreted with caution. Third, we were unable to include information on certain clinical variables known to be associated with differential prognosis in HF, such as LV ejection fraction, NYHA functional class, six-minute walking capacity, and other variables. Inclusion of this information may have better characterized severity of illness and risk of death for patients. In an attempt to address this concern, we did perform a subset analysis using available laboratory data for BNP, BUN, and sodium. Results of these analyses were similar to our main analyses. Finally, our analyses were not able to examine mediating factors contributing to the phenomenon of increased mortality among dual users.

While our observation of increased mortality risk among Veterans with HF who are dual users will be of interest to the VHA, we expect these findings will be of interest to clinicians and healthcare administrators outside the VHA as well. Both groups of facilities are interested in providing high quality care, and both groups are subject to quality measurement and reporting for 30-day all-cause mortality among patients hospitalized for heart failure. Cross-system care also frequently occurs among non-Veterans, and similar risks may apply to fractured care outside VA. Within VA, the recent advent and implementation of the Veterans Access, Choice, and Accountability Act of 2014 has actually accelerated the use of non-VA care among Vetearns enrolled in VHA, so this area of research is timely from a policy perspective. ("'An Act To improve the access of veterans to medical services from the Department of Veterans Affairs, and for other purposes" (PL 113-146, 7 August, 2014)")

\section{Conclusions}

In this study, we observed that dual users had higher mortality risk both from entry into the study cohort and from their final hospitalization event. While increasing access to healthcare for Veterans through provision of community care will doubtless have positive impacts across a variety of conditions, care must be taken to prevent negative unintended consequences of increased cross-system care. Further study of this systems-related HF phenomenon is needed to better understand the mediators of higher mortality risk among dual users. Examination is warranted for other diseases and care settings where dual use is associated with higher rates of healthcare utilization and mortality. Finally, future work should focus on the development and testing of interventions to mitigate the risk of cross-system care.

\section{Competing Interests Statement}

The authors declare that there are no competing or potential conflicts of interest.

\section{References}

Adams, K. F., Jr., Fonarow, G. C., Emerman, C. L., LeJemtel, T. H., Costanzo, M. R., Abraham, W. T., . . Horton, D. P. (2005). Characteristics and outcomes of patients hospitalized for heart failure in the United States: rationale, design, and preliminary observations from the first 100,000 cases in the Acute Decompensated Heart Failure National Registry (ADHERE). American Heart Journal, 149(2), 209-216. https://doi.org/10.1016/j.ahj.2004.08.005

Asch, S. M., McGlynn, E. A., Hogan, M. M., Hayward, R. A., Shekelle, P., Rubenstein, L., . . Kerr, E. A. (2004). Comparison of quality of care for patients in the Veterans Health Administration and patients in a national sample. Annals of Internal Medicine, 141(12), 938-945. https://doi.org/10.7326/0003-4819-141-12-200412210-00010 
Blecker, S., Ladapo, J. A., Doran, K. M., Goldfeld, K. S., \& Katz, S. (2014). Emergency department visits for heart failure and subsequent hospitalization or observation unit admission. American Heart Journal, 168(6), 901-908 e901. https://doi.org/10.1016/j.ahj.2014.08.002

Axon, R. N., Gebregziabher, M., Everett, C. J., Heidenreich, P., \& Hunt, K. J. (2016) Dual health care system use is associated with higher rates of hospitalization and hospital readmission among veterans with heart failure. American Heart Journal, 174, 157-163. https://doi.org/10.1016/j.ahj.2015.09.023

Chaudhry, S. I., McAvay, G., Chen, S., Whitson, H., Newman, A. B., Krumholz, H. M., \& Gill, T. M. (2013). Risk factors for hospital admission among older persons with newly diagnosed heart failure: findings from the Cardiovascular Health Study. J Am Coll Cardiol, 61(6), 635-642. https://doi.org/10.1016/j.jacc.2012.11.027

Cheng, R. K., Cox, M., Neely, M. L., Heidenreich, P. A., Bhatt, D. L., Eapen, Z. J., . . Fonarow, G. C. (2014). Outcomes in patients with heart failure with preserved, borderline, and reduced ejection fraction in the Medicare population. American Heart Journal, 168(5), 721-730. https://doi.org/10.1016/j.ahj.2014.07.008

Cox, D. R. (1972). Regression models and life tables. J R Stat Soc Ser, 34, 187-220.

Goodman, D. C., Fisher, E. S., \& Chang, C.-H. (2011). After hospitalization: A Dartmouth Atlas report on post-acute care for Medicare beneficiaries. In K. K. Bronner (Ed.), The Dartmouth Atlas of Healthcare (Vol. 2011).

Goodman, D. C., Fisher, E. S., \& Chiang-Hua, C. (February, 2013). The Revolving Door: A Report on U.S. Hospital Readmissons. Robert Wood Johnson Foundation.

Helmer, D., Sambamoorthi, U., Shen, Y., Tseng, C. L., Rajan, M., Tiwari, A., . . Pogach, L. (2008). Opting out of an integrated healthcare system: dual-system use is associated with poorer glycemic control in veterans with diabetes. Primary care diabetes, 2(2), 73-80. https://doi.org/10.1016/j.pcd.2008.02.004

Hernandez, A. F., Greiner, M. A., Fonarow, G. C., Hammill, B. G., Heidenreich, P. A., Yancy, C. W., . . Curtis, L. H. (2010). Relationship between early physician follow-up and 30-day readmission among Medicare beneficiaries hospitalized for heart failure. JAMA, 303(17), 1716-1722. https://doi.org/10.1001/jama.2010.533

Jia, H., Zheng, Y., Reker, D. M., Cowper, D. C., Wu, S. S., Vogel, W. B., .. Duncan, P. W. (2007). Multiple system utilization and mortality for veterans with stroke. Stroke, 38(2), 355-360. https://doi.org/10.1161/01.STR.0000254457.38901.fb

Keating, N. L., Landrum, M. B., Lamont, E. B., Bozeman, S. R., Krasnow, S. H., Shulman, L. N., . . McNeil, B. J. (2011). Quality of care for older patients with cancer in the Veterans Health Administration versus the private sector: a cohort study. Annals of Internal Medicine, 154(11), 727-736. https://doi.org/10.7326/0003-4819-154-11-201106070-00004

Lin, D. Y., Wei, L. J., \& Ying, Z. (1993). Checking the Cox model with cumulative sums of Martingale-based residuals. Biometrika, 80, 557-572. https://doi.org/10.1093/biomet/80.3.557

Lloyd-Jones, D., Adams, R., \& M., C. (2008). Heart Disease and Stroke Statistics 2009 Update. A report from the American Heart Association Statistics Committee and Stroke Statistics Subcommittee. Circulation, 108, 191261.

Lupon, J., Vila, J., \& Bayes-Genis, A. (2015). Risk Prediction Tools in Patients With Heart Failure. JACC Heart Fail, 3(3), 267. https://doi.org/10.1016/j.jchf.2014.10.010

Maciejewski, M. L., Wang, V., Burgess, J. F., Jr., Bryson, C. L., Perkins, M., \& Liu, C. F. (2013). The continuity and quality of primary care. Medical Care Research and Review, 70(5), 497-513. https://doi.org/10.1177/1077558713495454

Nasir, K., Lin, Z., Bueno, H., Normand, S. L., Drye, E. E., Keenan, P. S., \& Krumholz, H. M. (2010). Is same-hospital readmission rate a good surrogate for all-hospital readmission rate? Medical Care, 48(5), 477-481. https://doi.org/10.1097/MLR.0b013e3181d5fb24

Quan, H., Sundararajan, V., Halfon, P., Fong, A., Burnand, B., Luthi, J. C., . . Ghali, W. A. (2005). Coding algorithms for defining comorbidities in ICD-9-CM and ICD-10 administrative data. Medical Care, 43(11), 1130-1139. https://doi.org/10.1097/01.mlr.0000182534.19832.83

Roger, V. L., Weston, S. A., Redfield, M. M., Hellermann-Homan, J. P., Killian, J., Yawn, B. P., \& Jacobsen, S. J. (2004). Trends in heart failure incidence and survival in a community-based population. JAMA, 292(3), 
344-350. https://doi.org/10.1001/jama.292.3.344

Ross, J. S., Keyhani, S., Keenan, P. S., Bernheim, S. M., Penrod, J. D., Boockvar, K. S., . . Siu, A. L. (2008). Dual use of Veterans Affairs services and use of recommended ambulatory care. Medical Care, 46(3), 309-316. https://doi.org/10.1097/MLR.0b013e31815b9db3

Ross, J. S., Mulvey, G. K., Stauffer, B., Patlolla, V., Bernheim, S. M., Keenan, P. S., \& Krumholz, H. M. (2008). Statistical models and patient predictors of readmission for heart failure: a systematic review. Archives of Internal Medicine, 168(13), 1371-1386. https://doi.org/10.1001/archinte.168.13.1371

Selim, A. J., Kazis, L. E., Rogers, W., Qian, S., Rothendler, J. A., Lee, A., . . Fincke, B. G. (2006). Risk-adjusted mortality as an indicator of outcomes: comparison of the Medicare Advantage Program with the Veterans' Health Administration. Medical Care, 44(4), 359-365. https://doi.org/10.1097/01.mlr.0000204119.27597.f1

Tarlov, E., Lee, T. A., Weichle, T. W., Durazo-Arvizu, R., Zhang, Q., Perrin, R., .. . Hynes, D. M. (2012). Reduced overall and event-free survival among colon cancer patients using dual system care. Cancer Epidemiol Biomarkers Prev, 21(12), 2231-2241. https://doi.org/10.1158/1055-9965.EPI-12-0548

Trivedi, A. N., \& Grebla, R. C. (2011). Quality and equity of care in the veterans affairs health-care system and in medicare advantage health plans. Medical Care, 49(6), 560-568. https://doi.org/10.1097/MLR.0b013e31820fb0f6

Veterans Access, Choice, and Accountability Act of 2014128 C.F.R.

Weeks, W. B., West, A. N., Wallace, A. E., \& Fisher, E. S. (2008). Comparing the characteristics, utilization, efficiency, and outcomes of VA and non-VA inpatient care provided to VA enrollees: a case study in New York. Medical Care, 46(8), 863-871. https://doi.org/10.1097/MLR.0b013e31817d92e1

Wolinsky, F. D., An, H., Liu, L., Miller, T. R., \& Rosenthal, G. E. (2007). Exploring the association of dual use of the VHA and Medicare with mortality: separating the contributions of inpatient and outpatient services. $B M C$ Health Serv Res, 7, 70. https://doi.org/10.1186/1472-6963-7-70

Wolinsky, F. D., Miller, T. R., An, H., Brezinski, P. R., Vaughn, T. E., \& Rosenthal, G. E. (2006). Dual use of Medicare and the Veterans Health Administration: are there adverse health outcomes? BMC Health Serv Res, 6, 131. https://doi.org/10.1186/1472-6963-6-131

\section{Copyrights}

Copyright for this article is retained by the author(s), with first publication rights granted to the journal.

This is an open-access article distributed under the terms and conditions of the Creative Commons Attribution license (http://creativecommons.org/licenses/by/4.0/). 